Dudman, W. F. (1960). J. gen. Microbiol. 22, 25-39

\title{
Cellulose Production by Acetobacter Strains in Submerged Culture
}

\author{
BY W. F. DUDMAN \\ Colonial Microbiological Research Institute, Port of Spain, Trinidad, West Indies
}

SUMMARY: The normally slow rates of growth and of cellulose synthesis by certain strains of Acetobacter in static culture were accelerated greatly when these strains were cultured in shaken, indented flasks or in stirred aerated fermenters, whereby the time factor was reduced from 8-4 weeks to 2-4 days. Optimum yields of cellulose from Acetobacter acetigenum strain EA-I and A. xylinum strain HCC B-155 were produced in shaken, indented flasks, in a medium of hydrolysed molasses which contained 4-5\% of sugar and about $0.05 \%$ of nitrogen (as ammonia). Comparison of eleven strains of cellulose-forming Acetobacter sp. in static and shaken culture showed that all produced less cellulose and all except two showed less growth in shaken culture. Yields of cellulose in stirred, aerated fermenters decreased with increasing air flow rates, although the growth level remained constant. The results suggest that increased aeration decreases the yield of cellulose by causing decreased synthesis of cellulose per unit cell weight, but the involvement of celluloseless mutants cannot be excluded. Addition of neutralizing agents to the fermenter cultures increased the yields of cellulose by the order of $50 \%$.

The growth of cellulose-producing Acetobacter strains is slow in static cultures, even in the most favourable media; long cultural periods from 10 days to 6 weeks have been used in studies on cellulose production (e.g. Brown, 1886; Tarr \& Hibbert, 1931; Kaushal \& Walker, 1951). Acetobacter acetigenum requires up to 9,18 or 40 days for growth and cellulose synthesis to reach completion in a favourable hydrolysed molasses medium containing respectively $1.5 \%, 4.0 \%$ or $7.9 \%$ sugar (Dudman, 1959a). A possible explanation of the slow growth of these strictly aerobic organisms is that the pellicle at the air/liquid interface forms an effective barrier between atmospheric oxygen on one side and the nutrients on the other, causing the rate of growth to be limited by the rates at which these penetrate the pellicle to the cells within. It has long been known that pellicles in Acetobacter cultures may be sunk by gentle agitation allowing the formation of fresh pellicles, a process that may be repeated until the nutrients in the medium are exhausted. Since the multiple pellicles obtained in such intermittently disturbed cultures are each thinner than the single pellicle produced by undisturbed cultures grown under the same conditions it is possible that cellulose synthesis may be stimulated by such manipulations. No quantitative examination of this has been reported.

An obvious way of accelerating the growth of these organisms would be by preventing the formation of the pellicle altogether, by growth in vigorously shaken or agitated cultures. Schramm \& Hestrin (1954) examined the behaviour of Acetobacter xylinum in shaken cultures, and found that although the formation of the pellicle was prevented, growth being obtained instead 
in the form of stellate bodies, cellulose production after 2 and 5 days growth was less than half that in static cultures grown in the same medium for the same time. They also found that growth under agitated conditions favoured the growth of mutants unable to synthesize cellulose and that prolonged culture or repeated subculture on the shaker led to complete failure to produce cellulose. It is possible, however, that other strains may be capable of sustained high cellulose yields in shaken culture.

In the present study the behaviour of Acetobacter acetigenum strain EA-I was examined in static culture with multiple pellicle formation and in shaken cultures grown in smooth-walled and indented flasks. Cellulose yields from shaken cultures of fifteen Acetobacter strains were compared and the strains with the most favourable characteristics used for detailed study in shaken flasks and in 10 l. fermenters.

\section{METHODS}

Organisms. In addition to the organisms previously used (Dudman, 1959a) four other strains of Acetobacter xylinum were included in the present work. Strains NRRL B-42, NRRL B-43 and NRRL B-975 were obtained from the culture collection of the Northern Utilization Research and Development Division, U.S. Department of Agriculture, Peoria, Illinois, through the courtesy of Dr W. C. Haynes. Strain HCC B-155 was isolated from bruised grapes by Miss M. B. Morris, Hankey Culture Collection, at this Institute.

Media. Details of the media used are given with the results. The hydrolysed molasses was prepared by the action of crude yeast invertase as described previously (Dudman, 1959a) or by hydrolysis with phosphoric acid. In the latter case the crude blackstrap molasses was diluted with 2 vol. of water and $\frac{1}{15}$ th volume, relative to the initial molasses, of syrupy phosphoric acid (sp.gr. 1.75) added. The solution was boiled for $1 \mathrm{hr}$., cooled and concentrated ammonia added to give the desired concentration of ammonia-N. The molasses solution was then further diluted to $10 \mathrm{vol}$. relative to the initial volume of molasses. Sodium hydroxide solution was used to adjust to $\mathrm{pH} 6$ and the solution clarified by centrifugation. The medium was finally diluted to give the desired final sugar concentration (usually $5 \%$ sugar, requiring an overall 17-fold dilution).

Antifoam. Alkaterge C (Commercial Solvents Corporation, Terre Haute, Indiana, U.S.A.), $30 \%(\mathrm{v} / \mathrm{v})$ in paraffin oil. Antifoam (0.05 ml.) was added to all the indented flasks after sterilization of the medium.

Culture methods. The indented flasks were $250 \mathrm{ml}$. conical flasks with three equally spaced horn-like indentations in the walls, projecting $1-1.5 \mathrm{~cm}$. inwards and located $2.5 \mathrm{~cm}$. from the bottom. The shaker used was a New Brunswick 'model V' rotary shaker. The $4 \mathrm{l}$. fermentations were carried out in 10 l. stainless steel fermenters made to the specifications of Chain, Paladino, Ugolini, Callow \& van der Sluis (1954), with baffles and a $2 \frac{1}{2}$ in. diameter impeller. The static and shaken experiments were carried out at room temperature $\left(26^{\circ}-29^{\circ}\right)$, while the 10 l. fermenters were immersed in a water bath with running tap water $\left(24^{\circ}-29^{\circ}\right)$ without temperature control. 
Analytical methods. The static cultures and shaken cultures in smooth-walled flasks were analysed by the methods described by Dudman (1959a). The shaken cultures grown in the indented flasks did not produce the usual pellicle and therefore required a different procedure for the determination of cellulose and weight of organism. Methods based on the observation that the pulp-like cellulose product was retained by sintered glass filters were used. As a routine step before filtration $1 \mathrm{ml}$. of $10 \%(\mathrm{w} / \mathrm{v})$ mercuric chloride solution was added to the cultures to inhibit cellulose synthesis during analysis; organisms from young active cultures were capable of rapid cellulose synthesis in filtrates when sugar was present. The shaken cultures were filtered through sintered glass and the filtrates kept for analysis.

The pulpy residues on the filters were washed thoroughly with water and transferred to conical flasks, where they were suspended in $\mathrm{N}-\mathrm{NaOH}$ and left overnight on the rotary shaker. The suspensions were filtered on sintered glass and the cellulose residues acidified with dilute acetic acid until permanently acid to methyl red. The cellulose was washed exhaustively with water and dried to constant weight at $105^{\circ}$. Cellulose obtained in this way contained $0 \cdot 5-0.9 \% \mathrm{~N}$, equivalent to $3 \cdot 1-5 \cdot 6 \%$ protein, indicating that it was about $95 \%$ pure.

Residual sugar was determined in the initial culture filtrates after centrifugation to remove organisms. Growth in the shaken cultures was estimated in one of two ways. At first a direct method (method A) was used in which organisms in the turbid filtrates and washings were estimated turbidimetrically; the organisms remaining in the cellulose shreds were estimated by nitrogen determinations in the alkaline extracts, as in the case of solid pellicles (Dudman, 1959a). The growth in the culture was obtained by adding the results of the separate determinations. Reproducible results were obtained with replicate cultures but the method is tedious and unsuitable for large numbers of cultures. This disadvantage was avoided in an indirect method (method B) in which growth was measured by difference between the residual soluble nitrogen and the total nitrogen initially present in the medium, on the assumption that soluble nitrogen removed from the medium during growth is converted wholly to cell nitrogen. This assumption has been shown to be correct for ammonia nitrogen in defined medium (Dudman, 1959 b). In a series of comparative determinations on 14 shaken cultures using both methods, growth estimated by method A was lower in 3 cultures (by about $10 \%$ ), identical in 2 cultures and higher in 9 cultures (by about $30 \%$ ) than found by method B.

The cultures in the $10 \mathrm{l}$. fermenters were analysed in essentially the same way as those grown in the indented flasks. Samples were removed at intervals for $\mathrm{pH}$, residual sugar and residual nitrogen determinations. The samples were centrifuged before analysis to removed organisms and cellulose shreds. Growth was estimated by method B. Cellulose yields were determined only at the end of the fermentation when the fermenters were opened. The cellulose masses were collected initially by pouring the cultures through layers of cotton gauze; further handling was by centrifugation. Where necessary the cellulose 
was macerated in a Waring blendor before washing exhaustively with water, treatment with normal sodium hydroxide, acidification and further washing. The cellulose was bleached with chlorine dioxide, and again washed exhaustively. The white purified cellulose was collected either on sintered glass funnels or in a small basket centrifuge and dried at $105^{\circ}$.

\section{RESULTS}

\section{Multiple pellicle formation in static culture}

The effect of multiple pellicle formation on growth and cellulose production by Acetobacter acetigenum strain EA-I was examined in $500 \mathrm{ml}$. cultures grown in Glaxo penicillin flasks (Clayton, Hems, Robinson, Andrews, Hunwicke, 1944) on the following medium (all $\%, w / v$ ): hydrolysed molasses (invertase), 8.2 \% total sugar; $\left(\mathrm{NH}_{4}\right)_{2} \mathrm{SO}_{4}, 0.8 \% ; \mathrm{KH}_{2} \mathrm{PO}_{4}, 0.3 \% ; \mathrm{MgSO}_{4} .7 \mathrm{H}_{2} \mathrm{O}, 0.2 \%$; Marmite, $0.5 \%$; adjusted to $\mathrm{pH}$ 5. After sterilization $1 \%(\mathrm{v} / \mathrm{v})$ ethanol was added. The flasks were inoculated with $1 \mathrm{ml}$. of 2-day culture and the cultures grown for 27 days.

In three flasks the pellicles were sunk by gentle agitation on the seventh and fourteenth days and fresh pellicles permitted to grow, giving three pellicles in each flask. In three other cultures the pellicles were removed aseptically after 7 days and a second pellicle permitted to grow. Undisturbed cultures which produced one thick pellicle were used for comparison. The successive pellicles in all the cultures were analysed separately. The removal of intermediate pellicles from the culture surface did not have any significant effect on the total cellulose yield or growth (Table 1).

\section{Growth in smooth-walled shaken flasks}

The influence of shaken culture in smooth-walled flasks on growth and cellulose production by Acetobacter acetigenum strain EA-I was examined, using the hydrolysed molasses medium described above, with $3.4 \%$ (w/v) total sugar. The cultures were grown in $100 \mathrm{ml}$. medium in $250 \mathrm{ml}$. flasks without indentations, inoculated with $0.25 \mathrm{ml}$. of 2-day culture, and cultured under three sets of conditions for comparison: (a) shaken at 224 rev./ min., (b) static, $(c)$ intermediate conditions in which the cultures were grown static for 7 days to allow pellicles to form and then put on the shaker for 20 days.

Growth in the shaken flasks in the early stages resembled the stellate irregular bodies described by Schramm \& Hestrin (1954), but by the seventh day these combined to form large single masses which on further growth developed firm smooth surfaces. After about 20 days each culture contained one large, smooth, wedge-shaped mass of cellulose +organisms. It was thus possible to analyse the growth obtained in these shaken cultures with the methods used for static cultures. The cultures grown under the static and shaken conditions were analysed after $7,18,20$ and 27 days incubation, but the cultures grown under the intermediate conditions were analysed only after 27 days. 


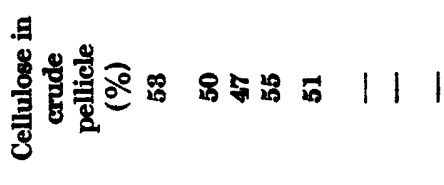

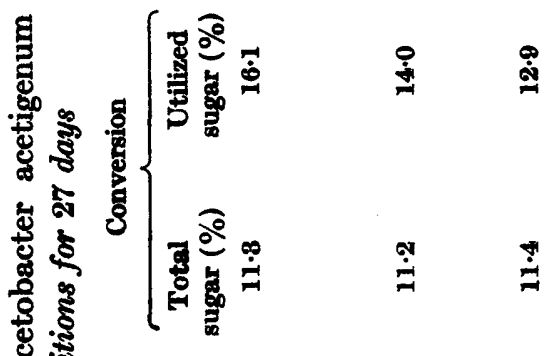

造

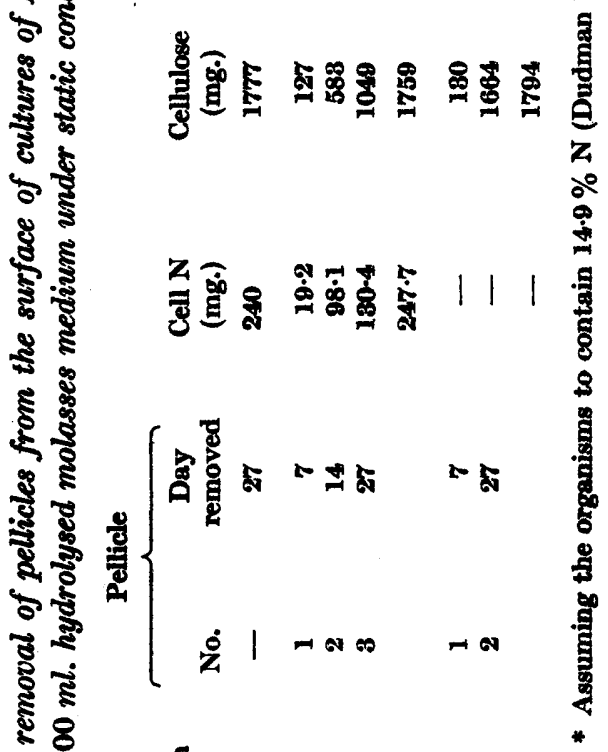

究

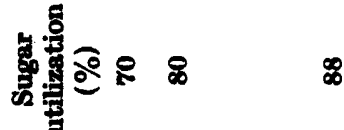

$\infty$

离

II $]$ 
The results are shown in Fig. 1. No important differences between the shaken and static cultures were found in the rates of final values of sugar utilization, cellulose production or growth. However, a difference between the shaken and static cultures was found in the pattern of conversion of utilized sugar. In the static cultures the conversion level was high (22-23\%) in the first half of the growth period and then decreased to half this level after 27 days. In fact cellulose synthesis had almost stopped during the last 7 days, while sugar utilization continued at an undiminished rate. Conversion of utilized sugar in the shaken flasks increased almost linearly during the first 20 days, after which it remained constant.
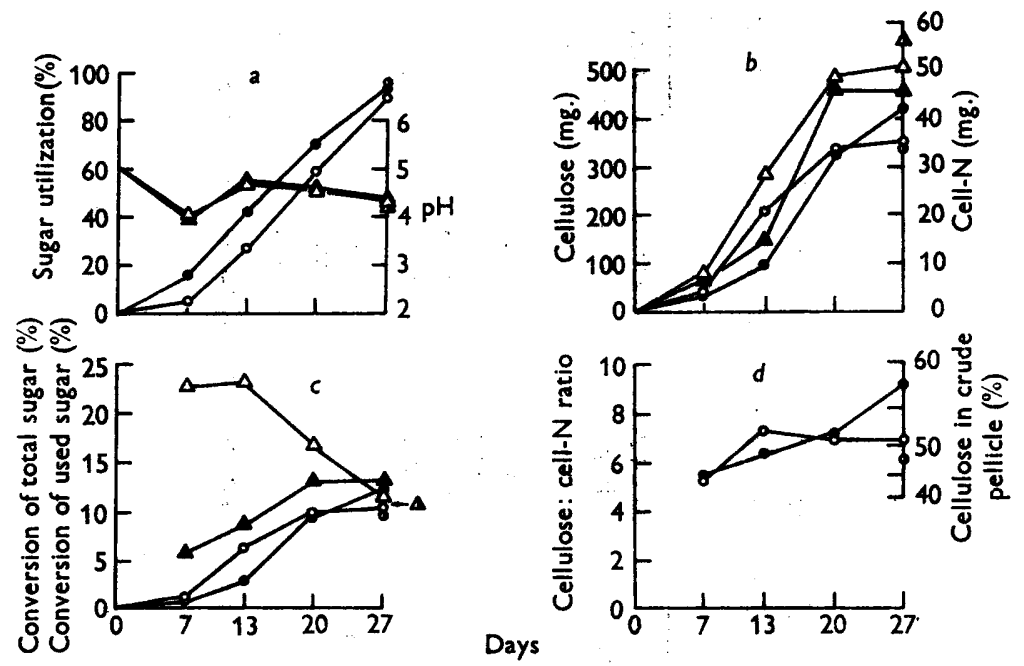

Fig. 1. Behaviour of Acetobacter acetigenum strain EA-I grown in $100 \mathrm{ml}$. hydrolysed molasses medium under static conditions $(O \Delta)$, in shaken smooth-walled flasks $(O \Delta)$, and under intermediate conditions (7 days static, 20 days shaken) ( $0 \Delta$ ). (a) Sugar utilization (\%) (O○O); pH ( $\Delta \Delta \Delta)$. (b) Cellulose (mg.) (O O ); cell-N (mg.) $(\Delta \Delta \Delta)$. (c) Conversion of total sugar (\%) (O○ ); Conversion of utilized sugar (\%) $(\Delta \Delta \Delta)$.

\section{Growth in indented shaken flasks}

Preliminary experiments with shaken cultures of Acetobacter acetigenum strain EA-I showed that indentation of the flasks prevented the formation of the large solid masses found in the shaken cultures grown in smooth-walled flasks. Growth was obtained in the form of the stellate bodies described by Schramm \& Hestrin (1954) even when the cultures were left on the shaker for 20 days, or when the organism was grown through six serial subcultures on the shaker.

The behaviour of this strain in shaken indented flasks was examined quantitatively in a hydrolysed molasses (invertase) medium containing (all $\%, w / v$ ) total sugar, $2.8 \% ;\left(\mathrm{NH}_{4}\right)_{2} \mathrm{SO}_{4}, 0.3 \% ; \mathrm{KH}_{2} \mathrm{PO}_{4}, 0.5 \%$; Marmite, $0.5 \%$; adjusted to $\mathrm{pH} 6 ; 100 \mathrm{ml}$. medium/flask. The flasks were inoculated with loopfuls from a sixth serial shaken subculture after $48 \mathrm{hr}$. growth. The cultures were grown on the shaker at $290 \mathrm{rev} . / \mathrm{min}$. and analysed by method $\mathrm{A}$. 
Sugar utilization, growth and cellulose synthesis were complete in 2 days (Fig. 2). The main activity occurred between 24 and $48 \mathrm{hr}$. Approximately equal weights of cellulose and organisms were synthesized by the cultures at all stages. The cellulose yield was equivalent to a conversion of 11-12\% of the total sugar available. Analysis of the washed organisms harvested from the 2-day cultures gave values of $3 \cdot 1 \%$ for total carbohydrate by the anthrone method and $13.7 \%$ for nitrogen by the micro-Kjeldahl method. This nitrogen value was used in all subsequent calculations of the dry weight of organisms.
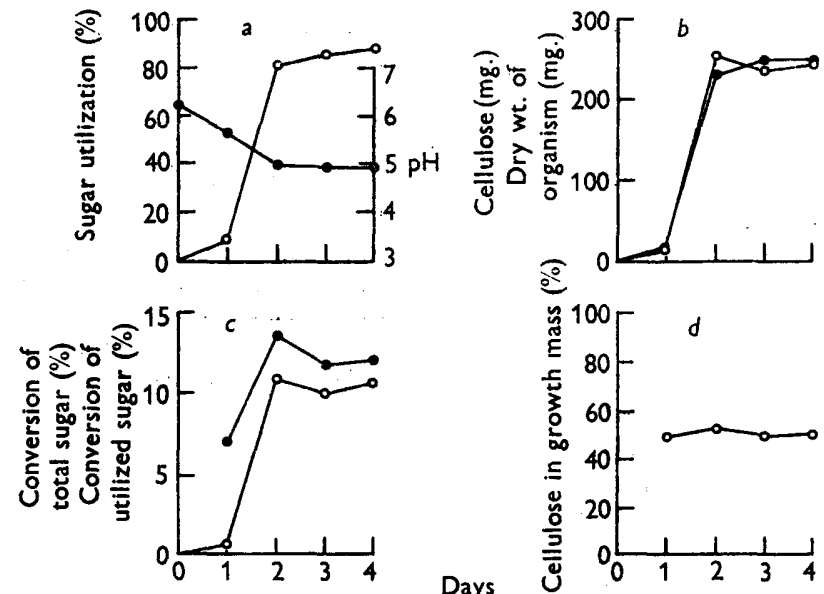

Days

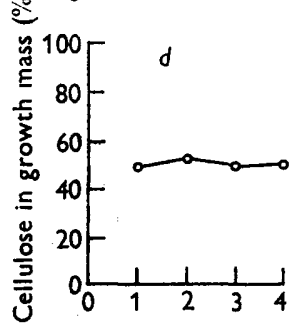

Fig. 2. Behaviour of Acetobacter acetigenum strain EA-I in $100 \mathrm{ml}$. hydrolysed molasses medium in shaken indented flasks. (a) Sugar utilization $(\%)(O) ; \mathrm{pH}(O)$. (b) Cellulose (mg.) (O); dry weight of organism (mg.) (O). (c) Conversion of total sugar (\%) (O); conversion of utilized sugar $(\%)(0)$.

\section{Comparison of strains in cultures grown in shaken indented flasks}

The behaviour of fifteen strains of Acetobacter organisms, capable of producing cellulose when grown under static conditions, was examined in shaken cultures grown in indented flasks. The organisms included Acetobacter acetigenum (2 strains), A. xylinum (10 strains), A. kützingianum (2 strains) and A. pasteurianum (1 strain), 11 strains of which had been examined in static culture previously (Dudman, 1959a). Each strain was examined in three serial cultures on the shaker to find whether cellulose yields were affected by repeated subculturing on the shaker.

The flasks, medium and shaking conditions used were the same as in the indented flask experiment above. The cultures were grown for 7 days and were analysed by method B. Sugar utilization was complete in all the cultures in 7 days. Four strains produced cellulose yields greater than $200 \mathrm{mg}$. in their first cultures, equivalent to conversion of $10-12 \%$ total sugar, but of these, only Acetobacter acetigenum strain EA-I consistently produced high yields in the subsequent cultures. Cellulose synthesis by the other highyielding strains (A. xylinum strains NRRL B-97.5, HCC B-155 and Hestrin) was reduced in the second cultures to the order of 150-165 mg., and in their 
third shaken cultures these strains produced yet smaller yields of the order of 180-140 mg. cellulose. All the other strains produced various smaller yields of cellulose in their first shaken cultures, with the exception of $A$. kützingianum strain Walker $\mathbf{D}$ which grew but produced no cellulose. Cellulose production by four of these strains was relatively constant, but cellulose yields from the others were progressively reduced in successive subcultures. Although the rates of growth and cellulose synthesis were increased by growing the shaken cultures in indented flasks, the figures in Table 2 show that when the results for static cultures (when available) are compared with those for the shaken cultures, all the strains produced less cellulose and all but two strains produced less growth on the shaker.

Table 2. Comparison of cell growth and conversion of total sugar into cellulose by various Acetobacter strains in static and shaken cultures grown in indented flasks

\begin{tabular}{|c|c|c|c|c|}
\hline \multirow[b]{2}{*}{ Organism } & \multirow[b]{2}{*}{ Strain } & \multicolumn{3}{|c|}{$\begin{array}{l}\text { Result of first shaken culture as percentage } \\
\text { of result from static culture* }\end{array}$} \\
\hline & & Cell-N & $\begin{array}{c}\text { Conversion of } \\
\text { total sugar }\end{array}$ & $\begin{array}{l}\text { Cellulose: } \\
\text { cell-N ratio }\end{array}$ \\
\hline - acetigenum & $\begin{array}{l}\text { EA-I } \\
\text { NCIB } 8182\end{array}$ & $\begin{array}{l}79 \\
78\end{array}$ & $\begin{array}{l}66 \\
58\end{array}$ & $\begin{array}{l}\mathbf{6 5} \\
\mathbf{5 8}\end{array}$ \\
\hline - xylinum & $\begin{array}{l}\text { HCC B-151 } \\
\text { HCC B-51 } \\
\text { Hestrin } \\
\text { Walker C } \\
472 \\
\text { HCC B-150 }\end{array}$ & $\begin{array}{r}76 \\
67 \\
79 \\
101 \\
69 \\
82\end{array}$ & $\begin{array}{l}\mathbf{5 7} \\
\mathbf{8 7} \\
\mathbf{4 3} \\
\mathbf{5 0} \\
\mathbf{8 0} \\
\mathbf{1 1}\end{array}$ & $\begin{array}{l}59 \\
43 \\
42 \\
39 \\
34 \\
10\end{array}$ \\
\hline ketitringianum & $\begin{array}{l}\text { Walker A } \\
\text { Walker D }\end{array}$ & $\begin{array}{l}54 \\
60\end{array}$ & $\begin{array}{r}78 \\
0\end{array}$ & $\underline{105}$ \\
\hline pasteurianum & Walker B & 124 & 78 & 47 \\
\hline
\end{tabular}

* Results for static cultures taken from Dudman (1959a).

Optimum sugar and nitrogen concentrations for shaken cultures

The influence of sugar concentration on cellulose yields from Acetobacter acetigenum strain EA-I and A. xylinum strain HCC B-155 in cultures grown in shaken indented flasks was examined in a medium containing hydrolysed molasses (invertase) at concentrations ranging from $0.9 \%$ to $10.7 \%$ total sugar and $\left(\mathrm{NH}_{4}\right)_{2} \mathrm{SO}_{4}, 0.3 \% ; \mathrm{KH}_{2} \mathrm{PO}_{4}, 0.5 \% ;$ Marmite $0.5 \%$ (all \%, w/v). The medium was adjusted to $\mathrm{pH}$. The flasks were inoculated with $1 \mathrm{ml}$. of 3-day static culture, and the cultures grown for 7 days in the shaker at 290 rev./ min.

Sugar concentrations below $\mathbf{3} \cdot 7 \%$ limited cellulose yields from both strains (Fig. 3); concentrations above $3.7 \%$ reduced the yields from strain EA-I, but the yields from strain HCC B-155 were unaffected by concentrations up to $10.7 \%$. However, the conversion of sugar to cellulose decreased with increasing sugar concentration. The two other high-yielding strains (Hestrin and NRRL-B-975) gave results very similar to strain EA-I. When examined at the other shaker speeds the same pattern of results was obtained. 
The influence of nitrogen concentration on cellulose production by strains EA-I and HCC B-155 was studied in a simplified medium omitting $\mathrm{KH}_{2} \mathrm{PO}_{4}$ and Marmite. The molasses was hydrolysed with phosphoric acid as described in Methods and various amounts of concentrated ammonia added to portions of the molasses to give concentrations ranging from $0 \%$ to $0 \cdot 106 \% \mathrm{~N}$ (as ammonia). The sugar concentration in the medium was $5 \cdot 3 \%(w / v)$. After inoculation with loopfuls of 4-day shaken cultures the cultures were grown for 5 days on the shaker at $290 \mathrm{rev}$./min. Cellulose yields from strain EA-I
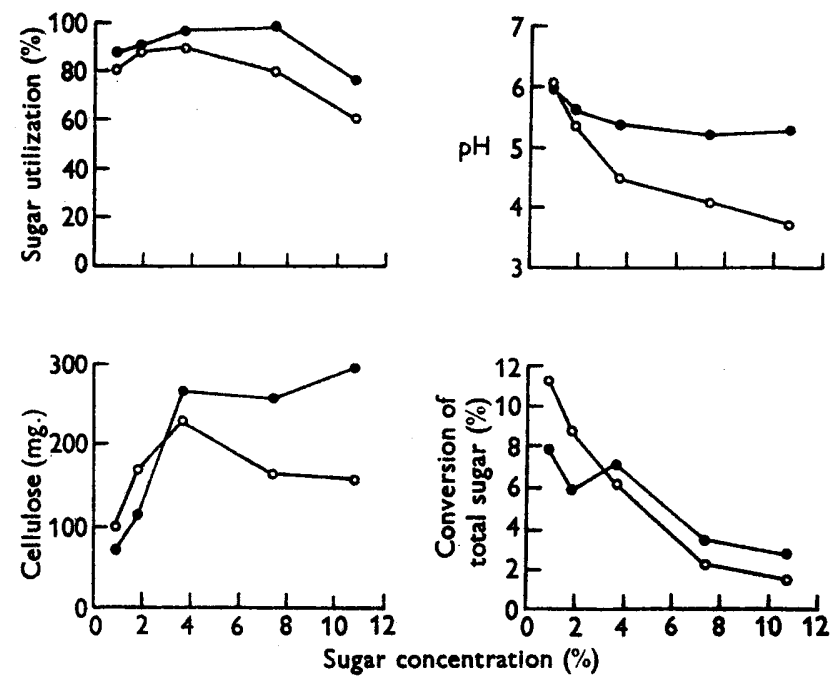

Fig. 3. Influence of sugar concentration in hydrolysed molasses medium on behaviour of $A$ cetobacter acetigenum strain EA-I (O) and $A$. aylinum strain HCC B-155 (O). $100 \mathrm{ml}$. cultures grown in shaken indented flasks (290 rev./min.) for 7 days.
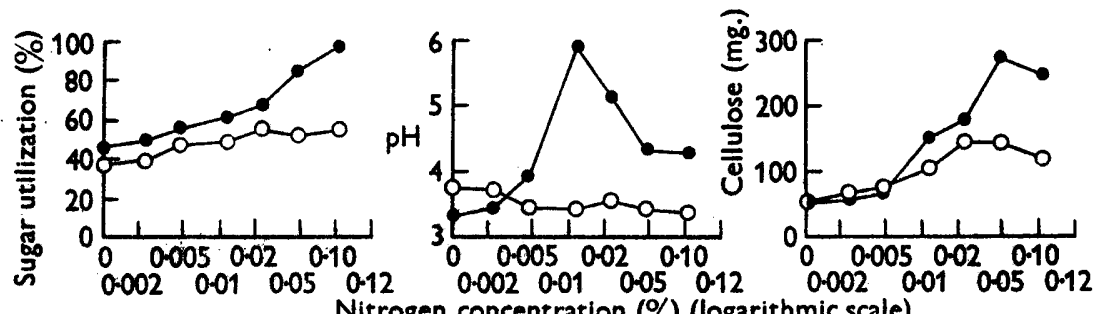

Nitrogen concentration (\%) (logarithmic scale)

Fig. 4. Influence of ammonia $\mathbf{N}$ concentration in hydrolysed molasses medium on behaviour of Acetobacter acetigenum strain EA-I (O) and A. xylinum strain HCC B-155 (O). 100 ml. cultures grown in shaken indented flasks (290 rev./min.) for 5 days.

were limited by nitrogen concentrations below $0.023 \%$ while yields from strain HCC B-155 were limited by concentrations below 0.046\% (Fig. 4). Both strains showed signs of sensitivity to higher nitrogen concentrations by giving slightly decreased yields at $0 \cdot 106 \% \mathrm{~N}$. As a result of these experiments it was decided to use the simplified medium containing 4-5\% sugar and 0.5$0.1 \% \mathrm{~N}$ (as ammonia) in subsequent submerged aerated cultures. 


\section{Submerged aerated cultures in $10 \mathrm{l}$. fermenters}

The behaviour of Acetobacter acetigenum strain EA-I and A. xylinum strain HCC B-155 was compared in the fermenters, and the latter found to have an important advantage. In addition to giving comparable high yields, strain HCC B-155 showed little tendency to form solid masses of growth on the baffles and propeller shaft, in contrast to strain EA-I which did so readily: Strain HCC B-155 was therefore chosen for further study. However, the ability of this strain to produce its growth in the form of well-divided floccules appears to be a mutable property making it necessary to maintain a number of parallel cultures which are tested at intervals. Strain HCC B-155, like strain EA-I, was found to be unable to attack sucrose; it was thus necessary to hydrolyse the sucrose in molasses before use.
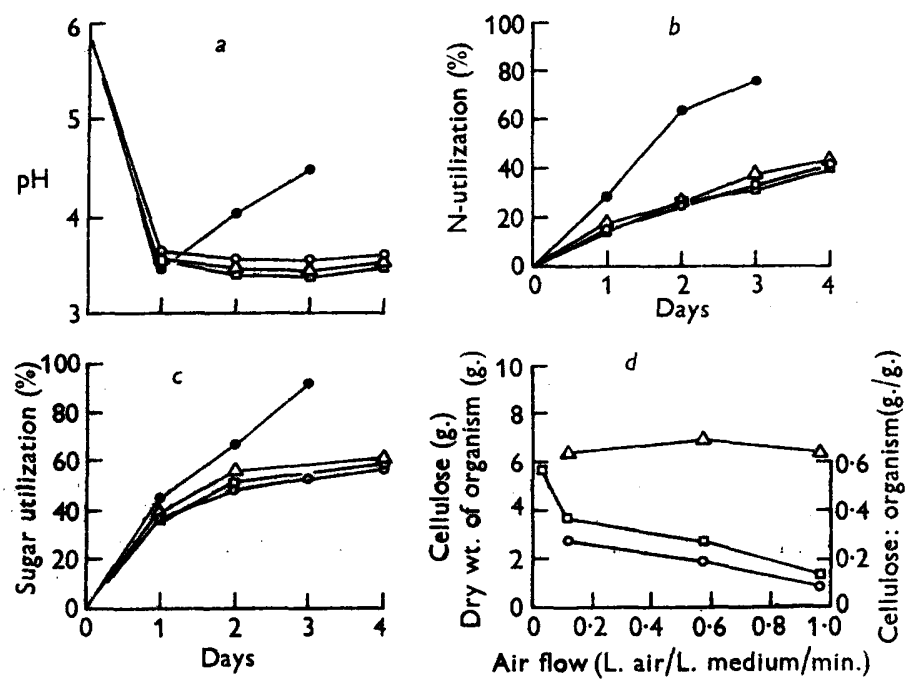

Fig. 5. Influence of air flow rate on behaviour of Acetobacter aylinum strain HCC B-155 grown in 41 , hydrolysed molasses medium in 101 . stirred (516 rev./min.) fermenters. $(a),(b),(c)$ Results for cultures aerated at $0.12,0.57$ and 0.971 . air $/ 1$. medium/min. shown as $O, \triangle$ and $\square$ respectively; results for $100 \mathrm{ml}$. cultures in shaken indented flasks shown as $\mathrm{O}$ (d) Cellulose (g.) (O); dry weight of organism (g.) $(\Delta)$; cellulose: organiam ratio (g./g.) ( $\square$ ). The result shown for 0.08 l. air $/ 1$. medium/min. was obtained with the control culture in the neutralization experiment (see text).

Aeration rate. The influence of the aeration rate on growth and cellulose yields was examined in cultures grown in the simplified medium containing $4.1 \%(w / v)$ total sugar and $0.08 \%(w / v) N$ (as ammonia). Each 10 l. fermenter was charged with 4 1. medium. After sterilization by autoclaving at $120^{\circ}$ for $45 \mathrm{~min}$. the medium was adjusted to $\mathrm{pH} 6$ by addition of $10 \%(\mathrm{w} / \mathrm{v})$ sodium hydroxide; $3 \mathrm{ml}$. of antifoam was added to each fermenter. The fermenters were inoculated with $200 \mathrm{ml}$. of 8-day sixth serial shaken culture of strain HCC B-155. The cultures were all stirred at $516 \mathrm{rev} . / \mathrm{min}$. but aerated at different rates $(0.12,0.57$ and 0.971 . air/l. medium/min.). Shortly after 
inoculation $100 \mathrm{ml}$. samples were removed aseptically in sterile indented flasks for growth on the shaker for comparison. At daily intervals shaken cultures and $50 \mathrm{ml}$. samples from the fermenters were analysed.

The rates of sugar and nitrogen utilization were not affected by the airflow rates used, but the rates in the fermenters were lower than in the shaken flasks (Fig. 5). Cellulose was produced by the cultures in the fermenters as a suspension of shreds or floccules consisting of the usual stellate bodies. Cellulose yields from the fermenters were decreased by increasing air flow rates, although the growth level was not affected. Increased aeration thus reduced the synthesis of cellulose/unit dry weight of organism. The most favourable conversion of total sugar to cellulose in the fermenters was only $1.5 \%$, while that in the shaken cultures was $\mathbf{3} \cdot 2 \%$.

Neutralizing agents. It was found in the experiment above that the $\mathrm{pH}$ values in the fermenters fell to about 3.5 within $24 \mathrm{hr}$. and showed no subsequent rise. It was thought possible that cellulose yields would be increased if the cultures were neutralized to keep the $\mathrm{pH}$ values more favourable. This possibility was explored by comparing results from a control culture with those from cultures neutralized by (i) daily addition of sodium hydroxide solution to adjust to $\mathrm{pH} \mathrm{6,} \mathrm{(ii)} \mathrm{daily} \mathrm{addition} \mathrm{of} 20 \mathrm{~g}$. chalk, and (iii) one addition of $80 \mathrm{~g}$. chalk at the beginning of the fermentation. Because the organism failed to grow in the medium when sterilized together with chalk it was necessary to sterilize the chalk separately; the evidence suggests that the inhibitory action of chalk sterilized in the medium is due to a $\mathrm{pH}$ effect.

The simplified medium was used, containing $4.4 \%(w / v)$ total sugar and $0.08 \% \mathrm{~N}$ (as ammonia). Each fermenter contained 41. medium. After sterilization two fermenters were adjusted to pH 6 with $22 \mathrm{ml} .10 \%(\mathrm{w} / \mathrm{v})$ $\mathrm{NaOH}$, while $80 \mathrm{~g}$. chalk and $20 \mathrm{~g}$. chalk were added to another two fermenters. Each fermenter was inoculated with $400 \mathrm{ml}$. of 3-day (first) shaken culture of strain HCC B-155. The cultures were stirred at $516 \mathrm{rev} . / \mathrm{min}$., and aerated at a very low rate $(0.03 \mathrm{l}$. air/l. medium/min.). At daily intervals $50 \mathrm{ml}$. samples were removed for analysis and the $\mathrm{pH}$ value in the fermenters adjusted by the appropriate treatment. The cultures were grown for 6 days.

Sugar utilization was more rapid in the neutralized cultures and reached higher final values than in the control culture (Fig. 6). Analysis of the residual sugar on the sixth day by a modification of the method of Mokrasch (1954) showed that $90 \%$ of the residual sugar in the control culture was fructose; the increased sugar utilization in the neutralized cultures was due to increased fructose utilization. When the cellulose was harvested, much of the chalk was found trapped in the cellulose shreds. Despite prolonged treatment with $10 \%(v / v)$ acetic acid, cellulose from the cultures neutralized with chalk had very high ash contents (Table B). When the results were corrected for ash, the cellulose yields from the three neutralized cultures were similar and represented an increase of the order of $50 \%$ over the control.

The value found for cellulose/dry wt. of organism in the control fermenter in the present experiment has been included in Fig. 5 for comparison with the values found in the aeration experiment. These results show that the high 


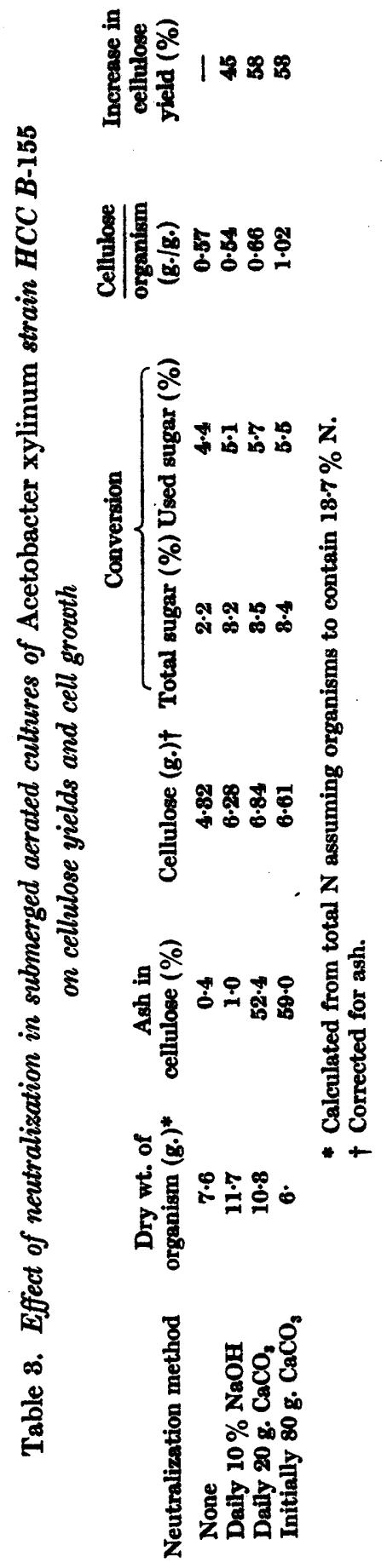


yreld of cellulose in the present control culture relative to those from the cultures in the aeration experiment arose from increased synthesis of cellulose/ unit dry weight of organism; the level of growth was of the same order in all these fermenters.
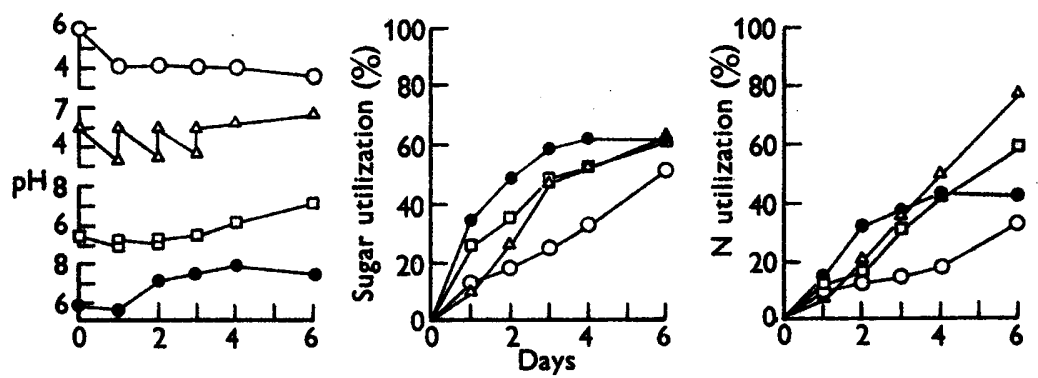

Fig. 6. Influence of neutralization on behaviour of Acetobacter xylinum strain HCC B-155 grown in 41 . hydrolysed molasses medium in 101 . stirred aerated fermenters (516 rev./ min.; 0.081 air/1. medium/min.). Results shown thus: untreated control culture (O); culture adjusted to pH 6 with 48, 81 and $25 \mathrm{ml} .10 \%(w / v) \mathrm{NaOH}$ after 1,2 and 8 days growth respectively $(\Delta)$; culture buffered with $20 \mathrm{~g}$. amounts of $\mathrm{CaCO}_{2}$ before inoculation and after 1 and 2 days growth ( $\square)$; culture buffered with $80 \mathrm{~g} \cdot \mathrm{CaCO}_{3}$ added before inoculation (O).

\section{DISCUSSION}

The slow rate of growth and cellulose synthesis of Acetobacter organisms was accelerated only when growth was carried out under conditions that prevented the formation of pellicles or large solid masses. The dependence of the increased growth rate on obtaining growth in a finely divided state, in which the effective surface area of the solid mass in contact with the medium is greatly increased, suggests that the slow growth rate under static conditions is caused by the slow rate of penetration of oxygen and nutrients into the pellicle. As growth and cellulose synthesis in static cultures take place mainly in the upper part of the pellicle (Schramm \& Hestrin, 1954) it is likely that the limiting process in static cultures is the rate of diffusion of oxygen into the pellicle.

Accelerated growth is obtained however at the expense of decreased cellulose yields. All the cellulose-producing strains for which static results are available produced lower yields in their first shaken cultures in indented flasks than in static cultures, and nearly all produced less growth. The decrease in cellulose synthesis, however, was relatively greater than that of growth, shown by the smaller cellulose:cell-nitrogen ratios obtained in the shaken cultures. The results obtained with the submerged aerated cultures of Acetobacter xylinum strain HCC B-155 clearly show the inverse relationship between aeration and cellulose yields, which was found to arise through decreased cellulose synthesis/unit of growth.

The inverse relationship between aeration and cellulose yields may be interpreted to mean that increased aeration leads to a shift in the metabolism of the organisms away from cellulose synthesis toward increased oxidation of the sugar substrate. Minor, Greathouse, Shirk, Schwartz \& Harris (1954) 
found evidence of this in static cultures of Acetobacter xylinum; they found that whenever oxidation of the substrate was of a high order, indicated by the $\mathrm{CO}_{2}$ produced, the yield of cellulose was usually small.

It is well recognized however that Acetobacter organisms readily mutate (Shimwell, 1956, 1957; Steel \& Walker, 1957b; Shimwell \& Carr. 1958), and it is well established that aerated cultural conditions favour the selection of celluloseless mutants of cellulose-producing strains (Schramm \& Hestrin, 1954; Wright \& Walker, 1955; Shimwell, 1956; Steel \& Walker, 1957a). As no attempt was made to examine any of the present cultures for celluloseless mutants it is not possible to decide to what extent the decreased yields found in the present aerobic cultures were due to conditions favouring the growth of mutants rather than to increased oxidation of the substrate. Possibly both mechanisms are involved, depending on the strain and cultural conditions. For example, it is thought unlikely that the selection of celluloseless mutants is responsible for decreased yields of cellulose from shaken cultures of strain EA-I, because the yield, although less, was constant through 6 successive shaken cultures. Celluloseless mutants are more likely to be involved where yields decrease in successive shaken cultures. Solution of this problem must await further studies on the influence of cultural conditions on cellulose yields in which celluloseless mutants and metabolic shifts are taken into account.

The author is indebted to Dr W. G. C. Forsyth, former Director of the Colonial Microbiological Research Institute, for his interest in this work, and to the Colonial Products Council for permission to publish.

\section{REFERENCES}

Brown, A. J: (1886). On an acetic ferment which forms cellulose. J. chem. Soc. p. 482. Chann, E. B., Paradino, S., Ugolini, F., Callow, D. S. \& van der Sluis, J. (1954). A laboratory fermenter for vortex and sparger aeration. R.C. Ist. sup. sanit. (English ed.), 17, 71.

Clayton, J. C., Heame, B. A., Robingon, F. A., Andrews, R. D. \& Humwicke, R. F. (1944). Preparation of penicillin. Improved method of isolation. Biochem.J. 38, 452.

Dudman, W. F. (1950a). Cellulose production by Acetobacter acetigenum and other Acetobacter species. J. gen. Microbiol. 21, 812.

Dudman, W. F. (1959b). Cellulose production by Acetobacter acetigenum in defined medium. J. gen. Microbiol. 21, 327.

KaUshat, R. \& Waxker, T. K. (1951). Formation of cellulose by certain species of Acetobacter. Biochem. J. 48, 618.

Mrnor, F. W., Greathouge, G. A., Shrrk, H. G., Schwartz, A. M. \& Harris, M. (1954). Biosynthesis of $\mathrm{C}^{14}$-specifically labelled cellulose by Acetobacter xylinum. II. From D-mannitol-1-C14 with and without ethanol. J. Amer. chem. Soc. 76, 5052.

Mokrasch, L. C. (1954). Analysis of hexose phosphates and sugar mixtures with the anthrone reagent. J. biol. Chem. $208,55$.

Schrasm, M. \& Husrrin, S. (1954). Factors affecting production of cellulose at the air/liquid interface of a culture of Acetobacter aylinum. J. gen. Microbiol. 11, 123.

Shmwerx, J. L. (1956). Transmutation of species in the genus Acetobacter. J. Inst. Brew. 62, 389. 
Shimwerl, J. L. (1957). A pattern of evolution in the genus Acetobacter. J. Inst. Brew. 63, 45.

ShImwELl, J. L. \& CARR, J. G. (1958). Old and new cellulose-producing Acetobacter species. J. Inst. Brew. 64, 47\%.

Steel, R. \& WALkER, T. K. $(1957 a)$. Celluloseless mutants of certain Acetobacter species. J. gen. Microbiol. 17, 12.

STEEL, R. \& WALKER, T. K. (1957b). Mutants of Acetobacter xylinum and Bacterium xylinoides which do not produce cellulose. J. Inst. Brew. 63, 510.

TARR, H. L. A. \& Hrbbert, H. (1981). Polysaccharide synthesis by the action of Acetobacter xylinus on carbohydrates and related compounds. Canad.J. Res. 4, 372.

Wright, H. B. \& Walker, T. K. (1955). Synthesis of melibiose by an Acetobacter species in a lactate-buffered glucose medium. Chem. \& Ind. (Rev.), 74, 18.

(Received 2 June 1959) 